Sepsis is a significant cause of morbidity and mortality and patients in the palliative phase of their illness are particularly vulnerable. In 2016 health care organisations were asked to review their management of the deteriorating (often septic) patient through a Patient Safety Alert from NHS Improvement.

Patients being cared for in hospice in- patient units are increasingly complex and earlier in their disease trajectory. This means that robust protocols must be in place to support staff in recognising acute deterioration, making an appropriate assessment and putting a management plan in place that takes account of the particular clinical complexities of the patient and their wishes and preferences with regard to care and treatment, including transfer to an acute setting if appropriate.

Hospital scoring systems and management approaches are often inappropriate in hospice in-patient units and it was with this in mind that we decided to develop our own protocol for care of the septic patient

This poster describes the development of a 'Sepsis Care Bundle' for Willowbrook Hospice specialist palliative care inpatient unit and the education that underpinned this. The Care bundle was designed to be easily accessible to and followed by staff, including flow charts and aide memoires.

\section{P-166 FEELING DRAINED = FEELING BETTER! AUDIT OF HOSPICE PLEURAL ASPIRATION}

Karen Groves. Queenscourt Hospice, Southport, UK

10.1136/bmjspcare-2017-hospice. 191

Background Hospice pleural aspiration has been undertaken for the last 25 years, following training by local chest physician. When the requirement for ultrasound (US) guided aspiration was introduced, a US machine was purchased and staff training undertaken. The British Thoracic Society issued guidance for pleural drainage in 2003 and standards were adapted for use in respect of pleural aspiration in hospice.

Method Retrospective review of all pleural aspirations undertaken over a three year period drawn from electronic clinical record. Documentation of the procedure was audited against 20 standards identified.

Results 36 procedures identified. 14 of 20 standards were $100 \%$ met; two met in $97 \%$ and two in 94\%. Although pleural aspiration was always undertaken with a standard pleural aspiration kit with a small bore needle and 3 way tap, the documentation did not specifically say so. The consent form (one missing) did not contain the list of common complications, which were outlined on the leaflet about the procedure given to each patient. The procedure sometimes took place out of hours where symptom control demanded it. Post procedure - analgesia was not always prescribed, as it was often done as a day case and patients brought their own and observations were not recorded as per protocol in the two failed procedures.

Conclusions The template for procedure documentation has been adjusted to incorporate items which were not present; consent form and information leaflet rewritten to include same list of common complications; analgesia now routinely prescribed and offered, and use of patient's own is documented; procedure adjusted to ensure that post- procedure observations completed even when procedure failed; anticoagulant administration and blood results documentation improved; standard rewritten to ensure that out of hours procedures should continue to take place when required for symptom management, but undertaken by most experienced clinician.

\section{P-167 RELIEVING THE PRESSURE! HOSPICE PARACENTESIS AUDIT ABSTRACT}

${ }^{1}$ Karen Groves, ${ }^{2}$ Sophie Bunn. 'Queenscourt Hospice, Southport, UK; ${ }^{2}$ Sheffield University Medical School, Sheffield, UK

10.1136/bmjspcare-2017-hospice. 192

Background Paracentesis is commonly used for drainage of malignant ascites in the palliative setting with $90 \%$ of patients reporting improvement of symptoms following the procedure. Despite this, there is limited evidence surrounding best practice and there are currently no national paracentesis standards. Aims To assess whether paracentesis standards are being met in practice, to compare with the results of 2010 audit and identify areas for improvement.

Method A retrospective analysis of all paracentesis procedures carried out in 2015 was done by searching for and analysing procedures documented on SystmOne (patient electronic record).

Results 41 procedures were performed on 10 patients, with average of $83 \%$ of standards met. All procedures had informed consent, INR documented and were done with appropriate anaesthetic and equipment. 98\% were preceded by ultrasound, compared with $43 \%$ in 2010 . Fewer procedures met the standards regarding documentation of observations and drain removal. PRN analgesia was prescribed in $85 \%$ of cases. The main complication was leakage post -drain removal.

Conclusions Results demonstrate more procedures are being done, with ultrasound now being used regularly to identify a safe drainage site. Recommendations were made to improve documentation of the insertion, observations and drain removal by altering the paracentesis template on SystmOne. Further action is to discuss the need for hourly observations and to research and review prevention and management of leaking from the drain site.

\section{P-168 USE OF DIAGNOSTIC ULTRASOUND IN A HOSPICE AT HOME TEAM - A SERVICE EVALUATION}

Jana Jeyakumar. Princess Alice Hospice, Esher, UK

\subsection{6/bmjspcare-2017-hospice. 193}

Background Ultrasound is a diagnostic tool that can supplement clinical examination. Members of the Princess Alice Hospice medical team have attended a course which teaches clinicians how to use ultrasound to assess for the presence of ascites and whether the urinary bladder contains fluid. The hospice has purchased a portable ultrasound machine. 